\title{
ISOTERMAS E CALOR ISOSTÉRICO DAS SEMENTES DE PINHÃO-MANSO
}

\author{
Tarcísio Honório Chaves ${ }^{1}$, Osvaldo Resende ${ }^{2}$, Daniel Emanuel Cabral de Oliveira ${ }^{3}$, Thaís Adriana de Souza \\ Smaniotto $^{4}$, Kelly Aparecida Sousa ${ }^{5}$
}

\begin{abstract}
RESUMO
O presente trabalho foi conduzido no Laboratório de Pós-Colheita de Produtos Vegetais do Instituto Federal Goiano, Câmpus Rio Verde, com os objetivos de obter as isotermas de dessorção e os valores de calor isostérico integral de dessorção das sementes de pinhão-manso. Os frutos foram colhidos e, posteriormente, procedeu-se ao beneficiamento das sementes, obtendo-se teor de água inicial de $20,9 \%$ (b.s.). Em seguida, foram submetidas à secagem em estufa com ventilação de ar forçada a $40^{\circ} \mathrm{C}$ até atingir os teores de água de 13,$6 ; 11,1 ; 8,7 ; 6,4 \%$ (b.s.). As isotermas de dessorção das sementes de pinhão-manso foram obtidas pelo método estático indireto, sendo a atividade de água $\left(\mathrm{a}_{\mathrm{w}}\right)$ determinada por meio do equipamento Hygropalm Model Aw 1. Para o controle da temperatura, utilizou-se uma B.O.D., regulada a 10, 20, 30 e $40{ }^{\circ} \mathrm{C}$. Aos dados experimentais, foram ajustados os modelos matemáticos frequentemente utilizados para representação da higroscopicidade de produtos agrícolas. O modelo de Sigma Copace é o que melhor representa a higroscopicidade das sementes de pinhão-manso. O calor isostérico integral de dessorção aumenta com a redução do teor de água, sendo que os valores, para as sementes de pinhão-manso, na faixa de teor de água de 5,6 a $13,4 \%$ (b.s.), variam de 3035,61 a $2631,89 \mathrm{~kJ} \mathrm{~kg}^{-1}$.
\end{abstract}

Palavras-chave: Jatropha curcas L, atividade de água e higroscopicidade.

\section{ABSTRACT \\ ISOTHERMS AND ISOSTERIC HEAT OF JATROPHA SEEDS}

This study was conducted at the Postharvest Laboratory of Vegetable Products of the Instituto Federal de Ciência, Educação e Tecnologia Goiano - Câmpus Rio Verde, seeking to obtain the desorption isotherms and the values of isosteric heat of desorption of jatropha seeds. The fruits were harvested and then preceded to processing of the seeds which presented an initial moisture content of $20.9 \%$ (d.b.). They were then dried in an oven with forced air ventilation at $40{ }^{\circ} \mathrm{C}$ until the moisture content of $13.6,11.1,8.7,6.4 \%$ (d.b.). The desorption isotherms of jatropha seeds were obtained by the indirect static method, and the water activity (aw) determined using the Hygropalm Aw Model 1 device. For temperature control a B.O.D was used, set at 10, 20,30 and $40{ }^{\circ} \mathrm{C}$. Experimental data was fitted to mathematical models often used to represent the hygroscopicity of agricultural products. The Sigma Copace model is that which best represents the hygroscopicity of jatropha seeds. The integral isosteric heat of desorption increased with decreasing moisture content, and the values for the jatropha seed in the moisture content range from 5.6 to 13.4 $\%$ (d.b.) varied from 3035.61 to $2631.89 \mathrm{~kJ} \mathrm{~kg}^{-1}$.

Keywords: Jatropha curcas L, water activity and hygroscopicity.

\section{Recebido para publicação em 08/05/2013. Aprovado em 23/05/2013.}

1 - Graduando em Agronomia no Instituto Federal de Educação, Ciência e Tecnologia Goiano - Câmpus Rio Verde, GO. IF Goiano, E-mail: tarcisiohonoriochaves@ymail.com Bolsista CNPq

2 - Eng. Agrícola, Prof. Dr. IF Goiano - Câmpus Rio Verde, E-mail: osvresende@yahoo.com.br

3 - Eng. Agrícola, Doutorando em Ciências Agrárias-Agronomia IF Goiano - Câmpus Rio Verde, E-mail: oliveira.d.e.c@gmail.com

4 - Eng. Agrônoma, Doutoranda em Ciências Agrárias-Agronomia IF Goiano - Câmpus Rio Verde, E-mail: tatiadris@ig.com.br

5 - Tecnóloga em Produção de Grãos, Doutoranda em Ciências Agrárias-Agronomia IF Goiano - Câmpus Rio Verde, E-mail: kellyapsousa@yahoo.com.br 


\section{INTRODUÇÃO}

A crescente preocupação mundial com o meio ambiente, juntamente com a busca por fontes de energia renováveis, coloca o biodiesel no centro das atenções e interesses. Diversos países, entre eles o Brasil, procuram o caminho do domínio tecnológico do biocombustível, tanto em nível agronômico como industrial, que deverá provocar fortes impactos na economia brasileira e na política de inclusão social do país (ABDALLA et al., 2008).

O Brasil é um país que apresenta grande potencial para se destacar na produção de biodiesel por dispor de uma grande extensão de áreas cultiváveis e clima adequado à produção de uma diversidade de culturas oleaginosas e, desta forma, disponibilizar matériaprima (SANTOS et al., 2009).

Os valores apresentados conferem ao óleo produzido de pinhão-manso os padrões estabelecidos pela ANP em sua especificação para óleos combustíveis, tanto para B100, ou seja, óleo diesel $100 \%$ vegetal, como para óleo diesel derivado de petróleo. As diversas características analisadas conferem ao óleo de pinhão diferenças em suas propriedades que o colocam como a oleaginosa mais promissora (BIODIESELBR, 2006). Souza et al. (2009) observaram uma concentração média

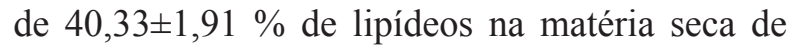
sementes de pinhão-manso.

Durante o armazenamento, podem ocorrer mudanças microbiológicas, químicas e físicas que, dependendo da interação entre estes fatores e o ambiente, podem ocasionar perdas de qualidade das sementes (RESENDE et al., 2006). Assim sendo, torna-se necessário o conhecimento das relações existentes entre as sementes de pinhãomanso, a temperatura, umidade relativa do ar e o teor de água do produto, visando amenizar possíveis alterações no material biológico.

Diversos pesquisadores têm estudado o equilíbrio higroscópico de diferentes produtos agrícolas, descrevendo modelos para expressar o teor de água de equilíbrio em função da temperatura e umidade relativa do ar. Para tal, são utilizados modelos matemáticos empíricos, uma vez que nenhum modelo teórico disponível tem sido capaz de predizer, com precisão, o teor de água de equilíbrio para uma ampla faixa de temperatura e umidade relativa do ar.
As isotermas podem ser definidas como curvas que descrevem a relação de equilíbrio de uma quantidade de água sorvida por componentes constituintes da semente e a umidade de equilíbrio, a uma temperatura específica. Sementes ricas em óleo apresentam teores de água de equilíbrio mais baixos em relação às sementes amiláceas, quando armazenadas em condições ambientais semelhantes, pois adsorvem menos água por serem hidrófobas (BROOKER et al., 1992).

As isotermas de sorção são importantes para definir limites de desidratação do produto, bem como estimar as mudanças de teor de água sob determinada condição de temperatura e umidade relativa do ambiente (AYRANCI; DUMAN, 2005). Todos os produtos agrícolas tem a propriedade de ceder ou absorver água do ambiente, convergindo para uma relação de equilíbrio entre o seu teor de água e as condições do ar ambiente. O teor de água de equilíbrio é alcançado quando a pressão parcial de vapor de água no produtor iguala-se à do ar que o envolve (RESENDE, 2006).

Ressalta-se ainda que a atividade da água é importante para o processamento de produtos agrícolas e sua conservação, pois se associa à atividade de água com a disponibilidade de água livre para o crescimento de microrganismos, além de outras reações que promovem a deterioração do produto (PARK et al., 2007).

Já o conhecimento do calor isostérico, em função do teor de água de equilíbrio, é essencial nos estudos de secagem e armazenamento de produtos vegetais, uma vez que a energia necessária para vaporizar é maior do que a utilizada para remover igual quantidade de água livre, nas mesmas condições de pressão e temperatura, constituindo, dessa forma, um bom parâmetro para estimar as necessidades energéticas no processo de secagem, além de fornecer dados sobre o estado da água no produto (WANG; BRENNAN, 1991).

Assim, objetivou-se, no presente trabalho, obter as isotermas de dessorção, bem como o calor isostérico integral de dessorção das sementes de pinhão-manso.

\section{MATERIAL E MÉTODOS}

O experimento foi conduzido no Laboratório de Pós-Colheita de Produtos Vegetais do Instituto 
Federal de Educação, Ciência e Tecnologia Goiano - Câmpus Rio Verde. Os frutos de pinhão-manso foram colhidos manualmente em janeiro de 2011, no município de Porangatu (GO). Posteriormente, procedeu-se ao a trilhagem manual das sementes, colocando-as expostas ao ambiente natural para reduzir o seu teor de água até 20,9\% (base seca, b.s.). Em seguida, foram submetidas à secagem em estufa com ventilação de ar forçada a $40{ }^{\circ} \mathrm{C}$ até atingir os teores de água de 13,$6 ; 11,1 ; 8,7 ; 6,4$ $\%$ (b.s.), determinados em estufa a $105 \pm 1{ }^{\circ} \mathrm{C}$, durante 24 h, em três repetições (BRASIL, 2009).
Para a obtenção dos dados das isotermas de dessorção das sementes de pinhão-manso, utilizouse o método estático indireto, sendo a atividade de água $\left(\mathrm{a}_{\mathrm{w}}\right)$ determinada por meio do equipamento Hygropalm Model Aw 1. Para o controle da temperatura, utilizou-se uma B.O.D., regulada a $10,20,30$ e $40^{\circ} \mathrm{C}$.

Aos dados experimentais foram ajustados aos modelos matemáticos frequentemente utilizados para representação da higroscopicidade de produtos agrícolas, cujas expressões estão descritas no Quadro 1.

Quadro 1. Modelos matemáticos utilizados para predizer a higroscopicidade de produtos agrícolas.

\begin{tabular}{|c|c|c|}
\hline Designação do modelo & Modelo & \\
\hline $\mathrm{Xe}^{*}=\exp \left\{\mathrm{a}-(\mathrm{b} \cdot \mathrm{T})+\left[\mathrm{c} \cdot \exp \left(\mathrm{a}_{\mathrm{w}}\right)\right]\right\}$ & Sigma Copace & (1) \\
\hline $\mathrm{Xe}^{*}=(\mathrm{a}+\mathrm{b} \cdot \mathrm{T}) /\left[\left(1-\mathrm{a}_{\mathrm{w}}\right) / \mathrm{a}_{\mathrm{w}}\right]^{\frac{1}{\mathrm{c}}}$ & Oswin Modificado & (2) \\
\hline $\mathrm{Xe}^{*}=\left[\ln \left(1-\mathrm{a}_{\mathrm{w}}\right) /(-\mathrm{a} \cdot(\mathrm{T}+\mathrm{b}))\right]^{\frac{1}{\mathrm{c}}}$ & Henderson Modificado & (3) \\
\hline $\mathrm{Xe}^{*}=\exp \left[\mathrm{a}-(\mathrm{b} \cdot \mathrm{T})+\left(\mathrm{c} \cdot \mathrm{a}_{\mathrm{w}}\right)\right]$ & Copace & (4) \\
\hline $\mathrm{Xe}^{*}=\mathrm{a}-\mathrm{b} \cdot \ln \left[-(\mathrm{T}+\mathrm{c}) \cdot \ln \left(\mathrm{a}_{\mathrm{w}}\right)\right]$ & Chung-Pfost & (5) \\
\hline $\mathrm{Xe}^{*}=\left[\exp (\mathrm{a}-\mathrm{b} \cdot \mathrm{T}) /-\ln \left(\mathrm{a}_{\mathrm{w}}\right)\right]^{\frac{1}{\mathrm{c}}}$ & Halsey Modificado & (6) \\
\hline $\mathrm{Xe}^{*}=\left[\ln \left(1-\mathrm{a}_{\mathrm{w}}\right) /(-\mathrm{a} \cdot(\mathrm{T}+273,16))\right]^{\frac{1}{\mathrm{~b}}}$ & Henderson & (7) \\
\hline$X e^{*}=a \cdot\left(\frac{a_{w}{ }^{b}}{T^{c}}\right)$ & Sabbah & (8) \\
\hline$X e^{*}=\left(a \cdot b \cdot c \cdot a_{w}\right) /\left[\left(1-c \cdot a_{w}\right) \cdot\left(1-c \cdot a_{w}+b \cdot c \cdot a_{w}\right)\right]$ & GAB & (9) \\
\hline $\mathrm{Xe}^{*}=\left[\log \left(1-\mathrm{a}_{\mathrm{w}}\right) /\left(\mathrm{a} \cdot\left(\mathrm{T}^{\mathrm{b}}\right)\right)\right]^{\frac{1}{\mathrm{c}}}$ & Cavalcanti Mata & $(10)$ \\
\hline
\end{tabular}

em que,

$\mathrm{Xe}^{*}=$ teor de água de equilíbrio, \% (b.s.);

$\mathrm{a}_{\mathrm{w}}=$ atividade de água, decimal;

$\mathrm{T}=$ temperatura, ${ }^{\circ} \mathrm{C}$; e

$\mathrm{a}, \mathrm{b}, \mathrm{c}=$ coeficientes. 
Os modelos matemáticos foram ajustados aos dados experimentais por meio de análise de regressão não linear pelo método Gauss-Newton, utilizando um programa estatístico. Os modelos foram selecionados considerando a magnitude do coeficiente de determinação $\left(\mathrm{R}^{2}\right)$, do teste de qui-quadrado $\left(\chi^{2}\right)$, do erro médio relativo $(\mathrm{P})$ e do desvio padrão da estimativa (SE). Considerou-se o valor do erro médio relativo inferior a $10 \%$ como um dos critérios para seleção dos modelos, de acordo com Mohapatra e Rao (2005).

$$
\begin{aligned}
& P=\frac{100}{N} \sum \frac{|\mathrm{Y}-\hat{\mathrm{Y}}|}{\mathrm{Y}} \\
& \mathrm{SE}=\sqrt{\frac{\sum(\mathrm{Y}-\hat{\mathrm{Y}})^{2}}{\mathrm{GLR}}} \\
& \chi^{2}=\frac{\sum(\mathrm{Y}-\hat{\mathrm{Y}})^{2}}{\mathrm{GLR}}
\end{aligned}
$$

em que,

$\mathrm{Y}=$ valor experimental;

$\hat{\mathrm{Y}}=$ valor estimado pelo modelo;

$\mathrm{N}=$ número de observações experimentais; e

GLR = graus de liberdade do modelo (número de observações experimentais menos o número de coeficientes do modelo).

Para os cálculos do calor isostérico líquido de sorção, para cada teor de água de equilíbrio, utilizou-se a equação de Clausius-Clayperon (IGLESIAS; CHIRIFE, 1976):

$$
\frac{\partial \operatorname{In}\left(\mathrm{a}_{\mathrm{w}}\right)}{\partial \mathrm{T}}=\frac{\Delta \mathrm{h}_{\mathrm{st}}}{\mathrm{RT}_{\mathrm{a}}^{2}}
$$

em que,

$\mathrm{a}_{\mathrm{w}}=$ atividade de água, decimal;

$\mathrm{T}_{\mathrm{a}}=$ temperatura absoluta, $\mathrm{K}$;

$\Delta \mathrm{h}_{\mathrm{st}}=$ entalpia diferencial, $\mathrm{kJ} \mathrm{kg}^{-1}$; e

$\mathrm{R}=$ constante universal dos gases, $8,314 \mathrm{~kJ} \mathrm{kmol}^{-1}$

$\mathrm{K}^{-1}=$ sendo para o vapor d'água $0,4619 \mathrm{~kJ} \mathrm{~kg}^{-1} \mathrm{~K}^{-1}$.

Integrando a Equação 14 e assumindo que o calor isostérico líquido de sorção seja independente da temperatura, o calor isostérico líquido de sorção, para cada teor de água de equilíbrio, foi obtido conforme a
Equação 15 (WANG; BRENNAN, 1991):

$\operatorname{In}\left(\mathrm{a}_{\mathrm{w}}\right)=-\left(\frac{\Delta \mathrm{h}_{\mathrm{st}}}{\mathrm{R}}\right) \cdot \frac{1}{\mathrm{~T}_{\mathrm{a}}}+\mathrm{C}$

em que,

$\mathrm{C}=$ coeficiente do modelo.

Os valores de atividade de água, temperatura e teor de água de equilíbrio foram obtidos a partir das isotermas de dessorção das sementes de pinhão manso, utilizando o modelo de Sigma Copace, o que melhor se ajustou aos dados experimentais.

O calor isostérico integral de sorção foi obtido adicionando-se, aos valores de calor isostérico líquido de sorção, o valor do calor latente de vaporização da água livre de acordo com a Equação 16.

$\mathrm{Q}_{\mathrm{st}}=\Delta \mathrm{h}_{\mathrm{st}}+\mathrm{L}=\mathrm{a} \cdot \exp \left(-\mathrm{b} \cdot \mathrm{Xe} \mathrm{e}^{*}\right)+\mathrm{L}$

em que,

$\mathrm{Q}_{\mathrm{st}}=$ calor isostérico integral de sorção, $\mathrm{kJ} \mathrm{kg}^{-1}$;

$\mathrm{L}=$ calor latente de vaporização da água livre, $\mathrm{kJ} \mathrm{kg}^{-1}$;

$\mathrm{Xe}^{*}=$ teor de água de equilíbrio, \% b.s.; e

$\mathrm{a}, \mathrm{b}=$ coeficientes do modelo.

O calor latente de vaporização da água livre $(\mathrm{kJ} /$ $\mathrm{kg}$ ) à temperatura de equilíbrio $\left({ }^{\circ} \mathrm{C}\right)$ foi calculado, utilizando-se a temperatura média $(\mathrm{T})$ na faixa em estudo, em ${ }^{\circ} \mathrm{C}$, por meio da seguinte equação:

$\mathrm{L}=2502,2-2,39 \cdot \mathrm{T}$

\section{RESULTADOS E DISCUSSÃO}

No Quadro 2, estão apresentados os parâmetros dos modelos de equilíbrio higroscópico para as sementes de pinhão-manso, obtidos por dessorção, para diferentes temperaturas.

Verifica-se que os modelos matemáticos, exceto GAB e Sabbah, utilizados para descrever a higroscopicidade das sementes de pinhão-manso apresentaram significância dos seus parâmetros de regressão ao nível de $1 \%$ de significância pelo teste t. Nota-se que os modelos exibiram elevados valores do coeficiente de determinação (superiores a $95 \%$ ), tendo o modelo Sigma Copace apresentado o maior valor $(99,45 \%)$. 
Entre os modelos analisados, o Sigma Copace apresentou menor erro médio estimado (SE), quiquadrado $\left(\chi^{2}\right)$ e valores do erro médio relativo (P) inferiores aos demais modelos e inferior a 10 $\%$, indicando uma representação adequada do fenômeno estudado, de acordo com Mohapatra e Rao (2005). Valores menores para SE permitem o bom ajuste dos modelos aos dados experimentais. A capacidade do modelo em descrever com fidelidade o processo físico é inversamente proporcional ao valor de SE (CHEN;JAYAS, 1998). Ressalva-se que os modelos apresentaram qui-quadrado calculado menor que o tabelado a 1 $\%$ de significância $\left(\chi_{\text {Tabelado }}^{2}=5,812\right)$.

Quadro 2. Parâmetros dos modelos de equilíbrio higroscópico para sementes de pinhão-manso, com seus respectivos erros médios estimados (SE) e relativo (P), qui-quadrado $\left(\chi^{2}\right)$ e coeficientes de determinação $\left(\mathrm{R}^{2}\right)$.

\begin{tabular}{|c|c|c|c|c|c|}
\hline Modelos & Parâmetros & SE (decimal) & $\mathrm{P}(\%)$ & $\chi^{2}($ decimal $)$ & $\mathrm{R}^{2}(\%)$ \\
\hline \multirow{3}{*}{ Sigma Copace } & $\mathrm{a}=0,2487 * *$ & 0,2214 & 1,9841 & 0,0490 & 99,45 \\
\hline & $\mathrm{b}=0,0047 * *$ & & & & \\
\hline & $\mathrm{c}=1,0348^{* *}$ & & & & \\
\hline \multirow{3}{*}{ Oswin Modificado } & $a=7,3486^{* *}$ & 0,3506 & 2,6761 & 0,1229 & 98,63 \\
\hline & $\mathrm{b}=-0,0351 * *$ & & & & \\
\hline & $\mathrm{c}=2,5341 * *$ & & & & \\
\hline \multirow{3}{*}{ Henderson Modificado } & $\mathrm{a}=0,0003 * *$ & 0,2836 & 2,4386 & 0,0804 & 99,11 \\
\hline & $\mathrm{b}=125,8063^{* *}$ & & & & \\
\hline & $\mathrm{c}=1,4287^{* *}$ & & & & \\
\hline \multirow{3}{*}{ Copace } & $\mathrm{a}=0,9026^{* *}$ & 0,2943 & 2,7703 & 0,0866 & 99,04 \\
\hline & $\mathrm{b}=0,0043 * *$ & & & & \\
\hline & $\mathrm{c}=2,0577^{* *}$ & & & & \\
\hline \multirow{3}{*}{ Chung-Pfost } & $\mathrm{a}=25,0273 * *$ & 0,3604 & 3,2994 & 0,1299 & 98,56 \\
\hline & $\mathrm{b}=4,3660^{* *}$ & & & & \\
\hline & $\mathrm{c}=79,4034 * *$ & & & & \\
\hline \multirow{3}{*}{ Halsey Modificado } & $\mathrm{a}=3,987476^{* *}$ & 0,4480 & 3,6324 & 0,2007 & 97,77 \\
\hline & $\mathrm{b}=0,012276^{* *}$ & & & & \\
\hline & $\mathrm{c}=2,154708^{* *}$ & & & & \\
\hline \multirow{2}{*}{ Henderson } & $\mathrm{a}=0,000161 * *$ & 0,3846 & 3,3062 & 0,1479 & 98,23 \\
\hline & $\mathrm{b}=1,447109 * *$ & & & & \\
\hline \multirow{3}{*}{ Sabbah } & $\mathrm{a}=19,57275^{* *}$ & 0,5636 & 5,6043 & 0,3176 & 96,47 \\
\hline & $\mathrm{b}=1,33144 * *$ & & & & \\
\hline & $\mathrm{c}=0,07471^{*}$ & & & & \\
\hline \multirow{3}{*}{ GAB } & $a=5,641336^{* *}$ & 0,6013 & 4,8262 & 0,3616 & 95,98 \\
\hline & $\mathrm{b}=4,830349^{\mathrm{ns}}$ & & & & \\
\hline & $\mathrm{c}=0,712798 * *$ & & & & \\
\hline \multirow{3}{*}{ Cavalcanti Mata } & $\mathrm{a}=-0,032429 * *$ & 0,3308 & 2,6183 & 0,1095 & 98,78 \\
\hline & $\mathrm{b}=0,136514^{* *}$ & & & & \\
\hline & $\mathrm{c}=1,433025^{*} *$ & & & & \\
\hline
\end{tabular}

** Significativos a 1\% de significância pelo teste t.

* Significativos a 5\% de significância pelo teste t.

${ }^{\text {ns }}$ Não significativo pelo teste t. 
Analisando estes parâmetros estatísticos, os modelos avaliados representam satisfatoriamente o equilíbrio higroscópico das sementes de pinhão-manso. Santos et al. (2010), estudando as isotermas de equilíbrio higroscópico das sementes de pinhão-manso comparando apenas os modelos de Henderson e Oswin, verificaram que o modelo de Henderson foi o que melhor representou as isotermas de dessorção do produto. Entretanto, o modelo Sigma Copace apresentou maior coeficiente de determinação e menores valores de $\chi^{2}, \mathrm{P}$ e SE, sendo recomendado para predição do fenômeno.

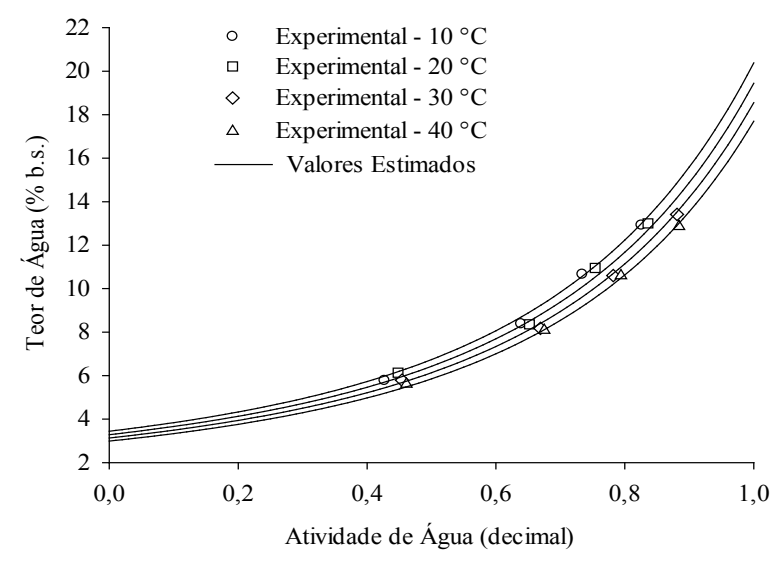

Figura 1. Isotermas de dessorção de água para as sementes de pinhão-manso, estimadas pelo modelo de Sigma Copace, para diferentes condições de temperatura e umidade relativa do ar.

Verifica-se, ainda, na Figura 1 que a as isotermas de dessorção das sementes de pinhãomanso são classificadas como do tipo III (IUPAC, 1985), assim como relatado para as sementes de nabo forrageiro (SOUSA et al., 2013), açaí e cupuaçu (SILVA et al., 2008). A atividade de água das sementes de pinhão-manso é proporcional ao teor de água, pois aumenta com a elevação do teor de água e temperatura. Este comportamento segue a mesma tendência da maioria dos produtos agrícolas.

As obtenções das isotermas auxiliam na identificação das condições de temperatura e umidade relativa do ar que podem favorecer o desenvolvimento de microrganismos ou secagem excessiva do produto, depreciando sua qualidade (CORRÊA et al., 2005). Assim, pelas isotermas obtidas, podem-se manejar adequadamente as sementes de pinhão-manso, visando à manutenção do seu teor de água em níveis seguros para o armazenamento, entre 7,0 e $8,0 \%$ (b.s.), controlando a atividade de água e minimizando, dessa forma, os processos que desencadeiam o desenvolvimento de microrganismos, insetos, bem como atividades metabólicas no produto. De acordo com Brooker et al. (1992), a faixa ótima para armazenamento de oleaginosas está entre 6,0 e $10,0 \%$ (b.s.).

Empregando-se as isotermas de sorção, podese também definir as regiões mais adequadas para o armazenamento das sementes de pinhão-manso, considerando os dados de temperatura e umidade relativa do ambiente.

Diante da representação satisfatória do equilíbrio higroscópico das sementes de pinhãomanso pelo modelo de Sigma Copace, calcularamse os valores de atividade de água necessários para a determinação dos valores do calor isostérico líquido de dessorção.

No Quadro 3, estão apresentados os valores de atividade de água estimados pelo modelo de Sigma Copace, para as temperaturas de 10, 20, 30 e $40{ }^{\circ} \mathrm{C}$ e para os teores de água de equilíbrio de 5,61 a $13,42 \%$ (b.s.). Nota-se que com aumento do teor de água e da temperatura ocorrre um acréscimo da atividade da água. Considerando que, em geral, os fungos têm o início do seu desenvolvimento com as atividades da água em torno de 0,7 (OLIVEIRA et al., 2005), verifica-se que o teor de água ideal para o armazenamento das sementes de pinhão manso está entre 8,0 e 7,0 $\%$ (b.s.), variando de acordo com a temperatura de armazenamento destas sementes.

$\mathrm{Na}$ Figura 2, são apresentadas as curvas do logaritmo neperiano da atividade da água para específicos valores de teor de água de equilíbrio (\% b.s.), em função de valores do inverso da temperatura absoluta $(\mathrm{K})$, para as sementes de pinhão-manso.

\section{REVENG}


Quadro 3. Valores da atividade da água (decimal) para as sementes de pinhão-manso estimados pelo modelo de Sigma Copace em função da temperatura e do teor de água.

\begin{tabular}{ccccc}
\hline Xe & \multicolumn{4}{c}{ Temperatura $\left({ }^{\circ} \mathrm{C}\right)$} \\
\cline { 2 - 5 } \% b.s. $)$ & 10 & 20 & 30 & 40 \\
\hline 5,61 & 0,3861 & 0,4165 & 0,4460 & 0,4747 \\
5,77 & 0,4044 & 0,4343 & 0,4633 & 0,4914 \\
5,79 & 0,4070 & 0,4368 & 0,4657 & 0,4938 \\
6,13 & 0,4432 & 0,4719 & 0,4999 & 0,5270 \\
8,09 & 0,6015 & 0,6261 & 0,6501 & 0,6735 \\
8,17 & 0,6073 & 0,6317 & 0,6556 & 0,6789 \\
8,36 & 0,6191 & 0,6432 & 0,6668 & 0,6899 \\
8,37 & 0,6195 & 0,6437 & 0,6672 & 0,6903 \\
10,59 & 0,7352 & 0,7567 & 0,7778 & 0,7985 \\
10,60 & 0,7356 & 0,7571 & 0,7782 & 0,7988 \\
10,65 & 0,7378 & 0,7593 & 0,7804 & 0,8010 \\
10,95 & 0,7504 & 0,7716 & 0,7924 & 0,8127 \\
12,86 & 0,8213 & 0,8411 & 0,8605 & 0,8795 \\
12,91 & 0,8230 & 0,8427 & 0,8621 & 0,8811 \\
13,00 & 0,8260 & 0,8457 & 0,8650 & 0,8839 \\
13,42 & 0,8391 & 0,8585 & 0,8776 & 0,8963 \\
\hline
\end{tabular}

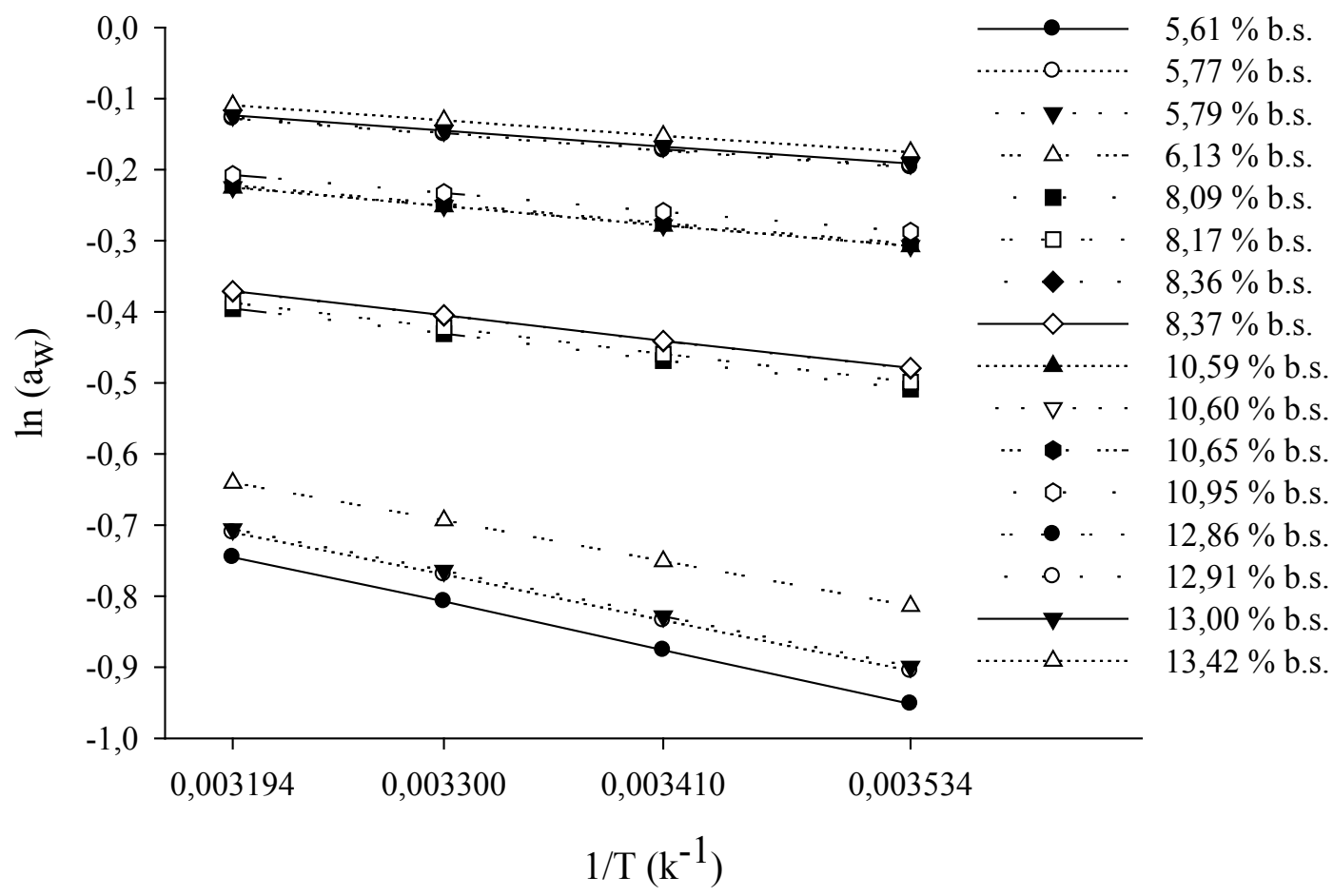

Figura 2. Curvas do logaritmo neperiano da atividade da água (decimal) para os valores de teor de água de equilíbrio (\% b.s.), em função de valores de $1 / \mathrm{T}\left(\mathrm{K}^{-1}\right)$, para sementes de pinhão-manso. 
Os valores do calor isostérico líquido de dessorção $\left(\mathrm{q}_{\mathrm{st}}\right)$, em $\mathrm{kJ} \mathrm{kg}^{-1}$, para as sementes de pinhão-manso, em função do teor de água de equilíbrio ( $\%$ b.s.), foram calculados de acordo com a Equação 15, representados, para cada situação, pelas inclinações das retas ilustradas na Figura 2. Para o calor isostérico integral de dessorção $\left(\mathrm{Q}_{\mathrm{s}}\right)$, em $\mathrm{kJ} \mathrm{kg}^{-1}$, foi adicionado aos valores de $\mathrm{q}_{\mathrm{st}} \mathrm{o}$ calor latente de vaporização da água livre (L), que representa a mínima quantidade de energia necessária para evaporar a água em estado livre, calculado para temperatura média de $25^{\circ} \mathrm{C}$, resultando em $2442,45 \mathrm{~kJ} \mathrm{~kg}^{-1}$.

Na Figura 3, está apresentada a curva do calor isostérico integral de dessorção, em função do teor de água de equilíbrio (\% b.s.), para as sementes de pinhão-manso.

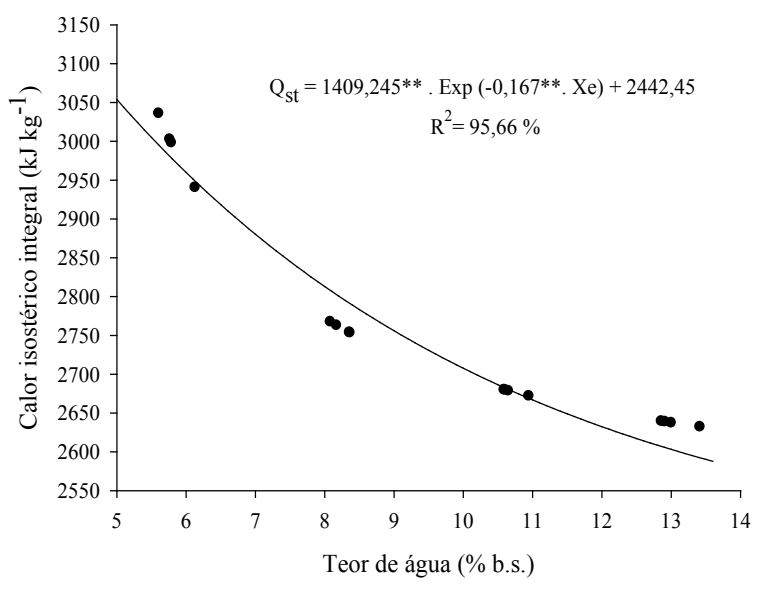

**Significativo a $1 \%$ pelo teste de $\mathrm{t}$.

Figura 3. Valores experimentais e estimados do calor isostérico integral de dessorção, em função do teor de água de equilíbrio, para as sementes de pinhão-manso.

Verifica-se que, com a redução do teor de água das sementes de pinhão-manso, ocorre aumento da energia necessária para a remoção de água do produto, representada pelos valores do calor isostérico integral de dessorção $\left(\mathrm{Q}_{\mathrm{st}}\right)$. Esse comportamento vem sendo observado por outros pesquisadores para diversos produtos agrícolas como feijão (RESENDE et al., 2006), pinhão (OLIVEIRA et al., 2010) e quinoa
(TOLABA et al., 2004).

Os valores do calor isostérico das sementes de pinhão-manso variaram de 3.035,61 a 2.631,89 $\mathrm{kJ} \mathrm{kg}^{-1}$ para a faixa de teor de água de 13,6 a 6,4 $\%$ (b.s.), respectivamente. Resende et al. (2006), estudando o calor isostérico integral de dessorção de grãos de feijão com teor de água de 21,7a 10,1 $\%$ (b.s.), verificaram valores superiores entre 3.961 e $2.718 \mathrm{~kJ} \mathrm{~kg}^{-1}$.

Para Brooker et al. (1992), é necessário uma quantidade de energia superior para remoção de água em produtos com baixo teor de água, quando se compara com produtos úmidos. De acordo com Al-Muhtaseb et al. (2004), isso ocorre porque na fase inicial de sorção (menor teor de água) existe uma camada monomolecular que necessita de elevadas quantidades de energia para remover essas moléculas de água do produto.

Ainda na Figura 3, observa-se que a equação de regressão exponencial estima o calor isostérico integral de dessorção para as sementes de pinhãomanso de forma satisfatória, pois apresenta elevado coeficiente de determinação $\mathrm{R}^{2}(95,66 \%)$, baixa magnitude do erro médio relativo $(0,90 \%)$ e erro médio estimado de 31,06 .

\section{CONCLUSÃO}

- A atividade de água das sementes de pinhãomanso é diretamente proporcional ao teor de água de equilíbrio higroscópico, sendo crescente com o aumento da temperatura, para o mesmo teor de água, seguindo a mesma tendência da maioria dos produtos agrícolas.

- O modelo de Sigma Copace é o que melhor representa a higroscopicidade das sementes de pinhão-manso.

- O calor isostérico integral de dessorção aumenta com a redução do teor de água, e os valores para as sementes de pinhão-manso na faixa de teor de água de 5,6 a 13,4\% (b.s.) variam de 3035,61 a $2631,89 \mathrm{~kJ} \mathrm{~kg}^{-1}$.

\section{AGRADECIMENTOS}

Ao $\mathrm{CNPq}$ e à Capes, pelo apoio financeiro indispensável na execução do presente trabalho. 


\section{REFERÊNCIAS BIBLIOGRÁFICAS}

ABDALLA, A.L.; SILVA FILHO, J.C.; GODOI, A.R.; CARMO, C.A.; EDUARDO, J.L.P. Utilização de subprodutos da indústria de biodiesel na alimentação de ruminantes. Revista Brasileira de Zootecnia, v.37, n. Especial, p.260-258, 2008.

AL-MUHTASEB, A.H.; MCMINN, W.A.M.; MAGGE, T.R.A. Water sorption isotherms of starch powders. Part 2: Thermodynamic characteristics. Journal of Food Engineering, v.62, n.2, p.135142, 2004.

AYRANCI, E.; DUMAN, O. Moisture sorption isotherms of cowpea (Vigna unguiculata L. Walp) and its protein isotate at 10,20 and $30^{\circ} \mathrm{C}$. Journal of Food Engineering, v.70, n.1, p.83-91, 2005.

BIODIESELBR, 2006. Disponível em: <http:// www.pinhaomanso.com.br/tudo-sobre-pinhaomansojatropha-curcas.html >. Acesso em: 07 ago. 2006.

BRASIL, Ministério da Agricultura e Reforma Agrária. Secretaria Nacional de defesa Agropecuária. Regras para Análise de Sementes. Brasília, 2009. 395p.

BROOKER, D.B.; BAKKER-ARKEMA, F.W.; HALL, C.W. Drying and storage of grains and oilseeds. New York: Van Nostran Reinhold, 1992. 450p.

CHEN, C.; JAYAS, D.S. Evaluation of GAB equation for the isoterms of agricultural products. Transactios of ASAE, v.41, n.6, p.1755-1760, 1998.

CORREAA, P. C.; RESENDE O.; RIBEIRO D. M. Isotermas de sorção das espigas de milho: obtenção e modelagem. Revista Brasileira de Milho e Sorgo, v.4, n.1, p.126-134, 2005.

IGLESIAS, H.; CHIRIFE, J. Prediction of the effect of temperature on water sorption isotherms of food material. International Journal of Science \& Technology, v.11, n.2, p.109-116, 1976.
IUPAC- International Union of Pure and Applied Chemistry. Reporting physisorption data for gas/ solid systems. Pure and Applied Chemistry, v. 57, n. 4, p. 603-619, 1985.

MOHAPATRA, D.; RAO, P.S. A thin layer drying model of parboiled wheat. Journal of Food Engineering, v.66, n.4, p.513-518, 2005.

OLIVEIRA, F. Adsorption isotherms of pinhão (Araucaria angustifolia seeds) starch and thermodynamic analysis. Journal of Food Engineering, v.100, p. 468-473, 2010.

OLIVEIRA, M.M.; CAMPOS, A.R.N.; GOMES, J.P.; SILVA, F.L.H. Isotermas de sorção do resíduo agroindustrial de casca do abacaxi (Ananas comosus L. Mer). Revista Brasileira de Engenharia Agrícola e Ambiental, v.9, n.4, p.565-569, 2005.

PARK, K.J.; ANTONIO, G.C.; OLIVEIRA, R.A.; PARK, K.J.B. Conceitos de processo e equipamentos de secagem. 121p. 2007. Disponível em: < http://www.feagri.unicamp.br/ ctea/manuais/concproceqsec_07.pdf $>$. Acesso em 06 de fev. 2012.

RESENDE, O. Variação das propriedades físicas e mecânicas e da qualidade do feijão (Phaseolus vulgaris L.) durante a secagem e armazenamento. 2006. 180f. Universidade Federal de Viçosa- Viçosa. - MG.

RESENDE, O.; CÔRREA, P.C.; GONELI, A.L.D.; RIBEIRO, D.M. Isotermas e calor isostérico de sorção do feijão. Ciência e Tecnologia de Alimentos, v.26, n.3, p.626-631, 2006.

SANTOS, S.B.; MARTINS, M.A.; CARVALHO, F.M.; CARNEIRO, A.C.O. Determinação de algumas propriedades físicas dos grãos de pinhão manso (Jatropha curcas L.) In: DI LEO, N.; MONTICO, S.; NARDÓN, G. Avances en Ingeniería Rural: 2007 - 2009. Rosario: UNR Editora, 2009, p.1067-1072.

SANTOS, S.B.; SAMPAIO, C.P.; MARTINS, M.A.; ARAÚJO, R.F. Isotermas de equilíbrio 
higroscópico para grãos de pinhão manso (Jatropha curcas L.) In: $4^{\circ}$ Congresso da Rede Brasileira de Tecnologia de Biodiesel e $7^{\circ}$ Congresso Brasileiro de Plantas Oleaginosas, Óleos, Gorduras e Biodiesel. Anais... Lavras: UFLA, 2010. v.1.

SILVA, A.E.; SILVA, L.H.M.; PENA, R.S. Comportamento higroscópico do açaí e cupuaçu em pó. Ciência e Tecnologia de Alimentos, v.28, n.4, p.895-901. 2008.

SOUSA, K.A. RESENDE, O.; COSTA, L.M. Isotermas de dessorção das sementes de nabo forrageiro obtidas pelos métodos dinâmico e estático. Revista Brasileira de Engenharia Agrícola e Ambiental, v. 17, n.2, p.216-222, 2013.
SOUZA, A.D.V.; FÁVARO, S.P.; ÍTAVO, L.C.V.; ROSCOE, R. Caracterização química de sementes e tortas de pinhão manso, nabo forrageiro e crambe. Pesquisa Agropecuária Brasileira, v.44, n.10, p.1328-1335, 2009.

TOLABA, M.P.; PELTZER, M.; ENRIQUEZ, N.; POLLIO, M.L. Grain sorption equilibria of quinoa grains. Journal of Food Engineering, v.61, n.3, p.365-371, 2004.

WANG, N.; BRENNAN, J.G. Moisture sorption isotherm characteristics of potato at four temperatures. Journal of Food Engineering, v.14, n.1, p.269-287, 1991. 